\title{
AVALIAÇÃO DO SOBREAQUECIMENTO DE ÓLEOS VEGETAIS ATRAVÉS DE ANÁLISES QUÍMICAS E ESPECTROSCOPIA UV/VISÍVEL
}

\author{
A. P. C. ARAÚJO' ${ }^{1}$, C. V. da $\operatorname{SILVA}^{1}$, A. F. G. MONTE${ }^{2}$ e F. R. X. BATISTA ${ }^{1}$ \\ ${ }^{1}$ Universidade Federal de Uberlândia, Faculdade de Engenharia Química \\ ${ }^{2}$ Universidade Federal de Uberlândia, Instituto de Física \\ E-mail para contato: frxbatista@feq.ufu.br
}

\begin{abstract}
RESUMO - Óleos vegetais representam um dos principais produtos extraídos das plantas e têm sido incluídos na dieta humana devido à sua larga utilização na preparação de alimentos. Contudo, o uso recorrente destes óleos leva à necessidade do monitoramento de seus parâmetros de qualidade, a fim de se verificar principalmente resistência e estabilidade à ação térmica. Assim, com o intuito de monitorar modificações potencialmente ocasionadas por elevadas temperaturas em amostras de óleo de soja, canola, milho e girassol, parâmetros como o índice de acidez, peróxido e iodo foram determinados através de análise físico-química, para diferentes temperaturas $\left(50\right.$ a $\left.150^{\circ} \mathrm{C}\right)$ e tempos de aquecimento (10 a $60 \mathrm{~min}$ ). Por meio de análises complementares de UV/vis foi possível também a determinação do estado oxidativo das amostras dos óleos. Os espectros de absorção obtidos mostraram que embora submetidas à ação térmica, o deslocamento batocrômico nas amostras de óleo não foi observado na região entre 200 a $400 \mathrm{~nm}$.
\end{abstract}

\section{INTRODUÇÃO}

Os óleos vegetais desempenham um papel importante na indústria de alimentos, melhorando as características sensoriais dos alimentos como sabor e textura. Os óleos vegetais são constituídos predominantemente por triglicerídios, e em menor quantidade, por outros constituintes de grande interesse, tais como tocoferóis, diglicerídios, ácidos graxos livres, esteróis, fosfolipídios e pigmentos (O’brien et al., 2000). A crescente utilização de óleos vegetais na dieta humana tem levado a um controle mais rigoroso deste produto, uma vez que óleos aquecidos e altamente oxidados podem apresentar substâncias tóxicas à saúde humana. Fatores como a incidência de luz, ação enzimática, exposição à atmosfera de oxigênio e elevadas temperaturas, comprometem a estabilidade química e física dos óleos durante seu processamento, armazenamento e uso, pois estão diretamente associados ao processo de oxidação do óleo. Durante a oxidação lipídica, modificações primárias podem ser detectadas pela perda dos ácidos graxos insaturados, além da formação de hidroperóxidos. Modificações secundárias podem ser monitoradas pela detecção da presença de compostos carbonil, aldeídos, hidrocarbonetos e produtos tóxicos (Almeida-Dória e Regitano-D’arce, 2000). É importante ressaltar que o processo de oxidação é acelerado ao se submeter o óleo ao sobreaquecimento, que 


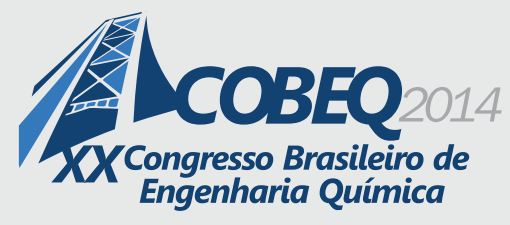

19 a 22 de outubro de 2014

Florianópolis/SC

resulta na degradação do óleo e consequentemente no comprometimento de suas propriedades. Neste contexto, faz-se necessário o uso de métodos analíticos capazes de medir a intensidade da degradação desta matéria prima e definir assim padrões de qualidade do óleo.

Dentre alguns métodos analíticos destacam-se o índice de acidez (AGL), o índice de peróxido (IPI) e o índice de iodo. O índice de acidez é definido como a quantidade em miligramas de hidróxido de potássio necessária para neutralizar os ácidos graxos livres de um grama da amostra do óleo (A.O.C.S, 2004). O AGL revela o estado de conservação do óleo, visto que a decomposição dos glicerídeos é acelerada pelo aquecimento e pela luz, sendo a rancidez quase sempre acompanhada pela formação de ácido graxo livre. A acidez, em geral, não é uma constante ou característica, mas uma variável relacionada com a natureza, qualidade da matéria-prima, grau de pureza, com o processamento e, principalmente, com as condições de conservação do óleo (Farhoosh et al., 2009). O IPI é um método clássico e sensível na determinação de hidroperóxidos. A presença destes compostos é um indício do início da deterioração das amostras de óleos e gorduras. Este índice é definido como a quantidade de peróxidos (expressa em miliquivalentes de oxigênio ativo por quilograma da amostra) que ocasiona a oxidação do iodeto de potássio, com a solução problema dissolvida em ácido acético e clorofórmio. Este índice fornece o grau de oxidação em que a gordura ou o óleo se encontram. É um método de titulação indireta, no qual o iodeto é oxidado a iodo pelos peróxidos liberados como subprodutos da oxidação lipídica. O iodo liberado é estequiometricamente relacionado ao teor de ácidos graxos. Já o índice de iodo é a medida do grau de insaturação de um óleo ou de uma gordura, ou seja, relaciona-se com a quantidade de duplas ligações presentes na amostra. Mais especificamente significa o número de gramas de iodo absorvido por $100 \mathrm{~g}$ de gordura ou óleo. Este parâmetro, portanto, proporciona a medida do grau de insaturação das gorduras extraídas com éter. Por essa razão, quanto maior a insaturação de um ácido graxo, maior será a sua capacidade de absorção de iodo e, consequentemente, maior será o índice (Moretto et al., 2002).

De forma complementar, a espectroscopia UV/vis é uma metodologia que tem sido frequentemente utilizada nas determinações analíticas em diversas áreas. Pode ser aplicada para determinações de compostos orgânicos e inorgânicos, sendo valiosa para a identificação dos grupos funcionais de uma molécula. A região ultravioleta é observada na faixa de 200 a $400 \mathrm{~nm}$, enquanto que a região do visível entre 400 a 800 nm (Silverstein et al., 1998). A absorção da região visível e ultravioleta depende, em primeiro lugar, do número e do arranjo dos elétrons nas moléculas ou íons absorventes. Como consequência, o pico de absorção pode ser correlacionado com o tipo de ligação que existe na espécie que está sendo estudada. Para os óleos vegetais, a presença de duplas ligações é verificada por um pico máximo de absorbância por comprimento de onda, característico de cada amostra. Essa verificação é possível devido à composição molecular homogênea dos óleos. Dessa forma, alterações na matéria prima como oxidação, podem ser identificadas pelo espectro de absorção, devido ao deslocamento de banda (efeito batocrômico) e o aumento da intensidade da absorção.

\section{MATERIAIS E MÉTODOS}

Os reagentes utilizados para a determinação dos índices de acidez e de peróxido foram todos de grau analítico e se resumiram a solução éter-álcool (2:1) neutra (v/v); solução de ácido acético- 
clorofórmio (3:2) (v/v); solução de hidróxido de sódio $(0,1 \mathrm{~N})$; solução de tiossulfato de sódio $(0,1 \mathrm{~N}$ e $0,01 \mathrm{~N})$; solução alcoólica de fenolftaleína; solução de iodeto de potássio $(1,4 \mathrm{~g} / \mathrm{mL})$; amido solúvel $(1 \% \mathrm{~m} / \mathrm{v})$ e água destilada (temperatura entre 5 e $\left.10^{\circ} \mathrm{C}\right)$. Para a determinação do índice de iodo utilizou-se solução etanólica de iodo $(0,1 \mathrm{~mol} / \mathrm{L})$, solução de tiossulfato de sódio $(0,1 \mathrm{~N})$ e solução saturada de amido. Os procedimentos experimentais foram realizados segundo a American Oil Chemists Society (1990) e o Instituto Adolfo Lutz (1985). O índice de acidez foi calculado através da Eq. 1.

$$
A G L(\%)=\left(V^{*} N^{*} f^{*} 100\right) / P
$$

$\mathrm{Na}$ qual $\mathrm{V}$ representa o volume $(\mathrm{mL})$ de hidróxido de sódio gasto na titulação; $\mathrm{N}$ a normalidade da solução de $\mathrm{NaOH}(0,1 \mathrm{~N}) ; f$ o fator de correção da solução de hidróxido de sódio $(0,945)$ e $\mathrm{P}$ a massa em gramas da amostra. O índice de peróxido foi obtido a partir da Eq. 2.

$$
\text { IPI }(\text { meq/1000g de amostra })=(V * N * f 1000) / P
$$

$\mathrm{Na}$ qual $\mathrm{V}$ representa o volume $(\mathrm{mL})$ da solução de tiossulfato de sódio gasto na titulação da amostra; $\mathrm{N}$ a normalidade da solução de tiossulfato de sódio $(0,1 \mathrm{~N}) ; f$ o fator da solução de tiossulfato de sódio $(1,18)$ e $\mathrm{P}$ a massa em gramas da amostra. Já o índice de iodo foi calculado pela Eq. 3.

$$
\text { Índice de Iodo }\left(g I_{2} / 100 g \text { de amostra }\right)=[(B-A) * C * f * 12,69] / M
$$

Sendo B o volume (mL) da solução de tiossulfato de sódio gasto na titulação da prova em branco; A o volume $(\mathrm{mL})$ da solução de tiossulfato de sódio gasto na titulação da amostra; C a concentração (mol/L) da solução de tiossulfato de sódio; $f$ o fator de correção da solução utilizada e M a massa em gramas da amostra. $\mathrm{O}$ cálculo quântico utilizado nas análises de espectroscopia UV/visível consistiu na determinação da quantidade de luz absorvida pela amostra $(A)$. Isto foi descrito pela lei de Beer- Lambert, representada pela Eq. 4, que dá a relação entre a intensidade da luz incidindo na solução $\left(I_{0}\right)$, e a intensidade da luz saindo da solução $(I)$. Além disso, tem-se a absorvidade molecular ou coeficiente de extinção $(\varepsilon)$, a concentração do material absorvedor $(c)$ e espessura da cubeta (caminho ótico) através da qual a luz percorre $(l)$. Para tal foram utilizadas cubetas de quartzo e espectrofotômetro da Ocean Optics modelo USB650, faixa 350-1000 nm, com fonte luminosa de tungstênio, halogênio e deutério.

$$
\log \left(I_{0} / I\right)=. A .=. \varepsilon . c . l
$$

Todos os parâmetros foram determinados para amostras comerciais dos óleos de soja, girassol, milho e canola, sendo estes submetidos às temperaturas de 50, 100 e $150^{\circ} \mathrm{C}$ e ambiente, após um período de exposição de 10, 15, 20 e 60 min. As determinações experimentais foram realizadas em triplicatas. 


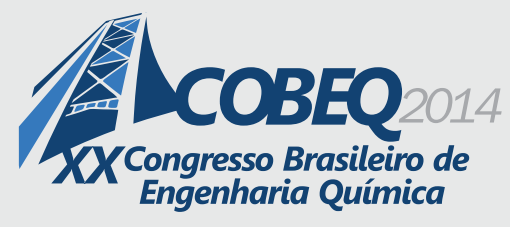

\section{RESULTADOS E DISCUSSÃO}

As Tabelas de 1 a 4 sumarizam os resultados obtidos para as análises físico-químicas, que abrangem AGL, IPI e índice de iodo de amostras de óleo de soja, milho, girassol e canola.

Tabela 1 - AGL, IPI e Índice de iodo do Óleo de Soja

\begin{tabular}{|c|c|c|c|}
\hline $\begin{array}{c}\text { Tempo / } \\
\text { Temperatura }\end{array}$ & $\begin{array}{c}\text { AGL } \\
(\%)\end{array}$ & $\begin{array}{c}\text { IPI } \\
(\mathrm{meq} / 1000 \mathrm{~g})\end{array}$ & $\begin{array}{c}\text { Iodo } \\
\left(\mathrm{g} \mathbf{I}_{2} / \mathbf{1 0 0 g}\right)\end{array}$ \\
\hline Controle & 0,542 & 4,66 & 139,907 \\
\hline $10 \mathrm{~min} / 50^{\circ} \mathrm{C}$ & 0,756 & 4,72 & 135,909 \\
\hline $15 \mathrm{~min} / 50^{\circ} \mathrm{C}$ & 0,945 & 4,72 & 129,247 \\
\hline $20 \mathrm{~min} / 50^{\circ} \mathrm{C}$ & 1,134 & 4,76 & 125,250 \\
\hline $10 \mathrm{~min} / 100^{\circ} \mathrm{C}$ & 0,378 & 7,08 & 131,912 \\
\hline $15 \mathrm{~min} / 100^{\circ} \mathrm{C}$ & 0,567 & 7,08 & 127,252 \\
\hline $20 \mathrm{~min} / 100^{\circ} \mathrm{C}$ & 0,756 & 9,44 & 112,268 \\
\hline $60 \mathrm{~min} / 100^{\circ} \mathrm{C}$ & 0,828 & 7,01 & 113,584 \\
\hline $10 \mathrm{~min} / 150^{\circ} \mathrm{C}$ & 0,567 & 4,72 & 129,247 \\
\hline $15 \mathrm{~min} / 150^{\circ} \mathrm{C}$ & 0,378 & 4,76 & 95,936 \\
\hline $20 \mathrm{~min} / 150^{\circ} \mathrm{C}$ & 0,283 & 5,50 & 85,276 \\
\hline
\end{tabular}

Tabela 3 - AGL, IPI e Índice de iodo do Óleo de Girassol

\begin{tabular}{|c|c|c|c|}
\hline $\begin{array}{c}\text { Tempo / } \\
\text { Temperatura }\end{array}$ & $\begin{array}{c}\text { AGL } \\
(\%)\end{array}$ & $\begin{array}{c}\text { IPI } \\
(\mathrm{meq} / \mathbf{1 0 0 0 g})\end{array}$ & $\begin{array}{cc}\text { Iodo } \\
\left(\mathrm{g} \mathrm{I}_{2} / \mathbf{1 0 0 g}\right)\end{array}$ \\
\hline Controle & 0,850 & 7,08 & 125,250 \\
\hline $10 \mathrm{~min} / 50^{\circ} \mathrm{C}$ & 0,756 & 7,08 & 122,585 \\
\hline $15 \mathrm{~min} / 50^{\circ} \mathrm{C}$ & 0,567 & 9,44 & 121,250 \\
\hline $20 \mathrm{~min} / 5^{\circ}{ }^{\circ} \mathrm{C}$ & 0,567 & 11,80 & 107,92 \\
\hline $10 \mathrm{~min} / 100^{\circ} \mathrm{C}$ & 0,535 & 6,49 & 119,920 \\
\hline $15 \mathrm{~min} / 100^{\circ} \mathrm{C}$ & 0,378 & 4,72 & 113,258 \\
\hline $20 \mathrm{~min} / 100^{\circ} \mathrm{C}$ & 0,425 & 4,70 & 102,598 \\
\hline $60 \mathrm{~min} / 100^{\circ} \mathrm{C}$ & 0,567 & 4,70 & 100,584 \\
\hline $10 \mathrm{~min} / 150^{\circ} \mathrm{C}$ & 0,378 & 9,44 & 121,250 \\
\hline $15 \mathrm{~min} / 150^{\circ} \mathrm{C}$ & 0,375 & 9,44 & 99,933 \\
\hline $20 \mathrm{~min} / 150^{\circ} \mathrm{C}$ & 0,285 & 10,62 & 97,268 \\
\hline
\end{tabular}

Tabela 2 - AGL, IPI e Índice de iodo do Óleo de Milho

\begin{tabular}{|c|c|c|c|}
\hline $\begin{array}{l}\text { Tempo / } \\
\text { Temperatura }\end{array}$ & $\begin{array}{l}\text { AGL } \\
(\%)\end{array}$ & $\begin{array}{c}\text { IPI } \\
(\mathrm{meq} / \mathbf{1 0 0 0 g})\end{array}$ & $\begin{array}{c}\text { Iodo } \\
\left(\mathrm{g} \mathrm{I}_{2} / \mathbf{1 0 0 g}\right)\end{array}$ \\
\hline Controle & 0,630 & 5,90 & 127,915 \\
\hline $10 \mathrm{~min} / 50^{\circ} \mathrm{C}$ & 0,945 & 4,72 & 105,26 \\
\hline $15 \mathrm{~min} / 50^{\circ} \mathrm{C}$ & 0,661 & 11,8 & 103,931 \\
\hline $20 \mathrm{~min} / 50^{\circ} \mathrm{C}$ & 0,756 & 10,22 & 101,266 \\
\hline $10 \mathrm{~min} / 100^{\circ} \mathrm{C}$ & 1,039 & 7,08 & 103,931 \\
\hline $15 \mathrm{~min} / 100^{\circ} \mathrm{C}$ & 1,134 & 9,44 & 99,933 \\
\hline $20 \mathrm{~min} / 100^{\circ} \mathrm{C}$ & 0,850 & 9,44 & 91,939 \\
\hline $60 \mathrm{~min} / 100^{\circ} \mathrm{C}$ & 0,976 & 7,94 & 97,216 \\
\hline $10 \mathrm{~min} / 150^{\circ} \mathrm{C}$ & 0,945 & 6,68 & 101,932 \\
\hline $15 \mathrm{~min} / 150^{\circ} \mathrm{C}$ & 0,567 & 7,86 & 98,601 \\
\hline $20 \mathrm{~min} / 150^{\circ} \mathrm{C}$ & 0,708 & 6,69 & 87,941 \\
\hline
\end{tabular}

Tabela 4 - AGL, IPI e Índice de iodo do Óleo de Canola

\begin{tabular}{|c|c|c|c|}
\hline $\begin{array}{c}\text { Tempo / } \\
\text { Temperatura }\end{array}$ & $\begin{array}{c}\text { AGL } \\
(\%)\end{array}$ & $\begin{array}{c}\text { IPI } \\
(\mathrm{meq} / 1000 \mathrm{~g})\end{array}$ & $\begin{array}{c}\text { Iodo } \\
\left(\mathrm{g} \mathrm{I} \mathrm{I}_{2} / \mathbf{1 0 0 g}\right)\end{array}$ \\
\hline Controle & 0,567 & 7,08 & 119,920 \\
\hline $10 \mathrm{~min} / 50^{\circ} \mathrm{C}$ & 0,630 & 7,12 & 118,588 \\
\hline $15 \mathrm{~min} / 50^{\circ} \mathrm{C}$ & 0,756 & 9,44 & 106,596 \\
\hline $20 \mathrm{~min} / 50^{\circ} \mathrm{C}$ & 0,630 & 11,01 & 93,271 \\
\hline $10 \mathrm{~min} / 100^{\circ} \mathrm{C}$ & 0,693 & 11,01 & 113,258 \\
\hline $15 \mathrm{~min} / 100^{\circ} \mathrm{C}$ & 0,819 & 11,80 & 95,936 \\
\hline $20 \mathrm{~min} / 100^{\circ} \mathrm{C}$ & 0,945 & 11,80 & 78,614 \\
\hline $60 \mathrm{~min} / 100^{\circ} \mathrm{C}$ & 0,401 & 8,41 & 97,810 \\
\hline $10 \mathrm{~min} / 150^{\circ} \mathrm{C}$ & 0,373 & 9,40 & 94,603 \\
\hline $15 \mathrm{~min} / 150^{\circ} \mathrm{C}$ & 0,382 & 9,44 & 87,941 \\
\hline $20 \mathrm{~min} / 150^{\circ} \mathrm{C}$ & 0,394 & 10,03 & 65,290 \\
\hline
\end{tabular}




\section{9 a 22 de outubro de 2014 \\ Florianópolis/SC}

É importante ressaltar que a oxidação é um processo autocatalítico e ocorre em aceleração crescente, uma vez iniciada. A oxidação lipídica envolve a formação contínua de hidroperóxidos como produtos primários que podem se decompor em uma variedade de produtos não voláteis e voláteis secundários. A taxa de formação de hidroperóxidos supera a sua taxa de decomposição durante o estágio inicial da oxidação e isto se reverte em estágios posteriores. Portanto, o IPI é considerado um indicador do estágio inicial de alterações oxidativas. A verificação da fase de crescimento ou diminuição da concentração de hidroperóxidos é possível através do monitoramento do tempo (Shahidi et al., 2005). Para interpretar os resultados dos testes de IPI, é importante ressaltar que os peróxidos são instáveis e formam componentes transitórios durante a oxidação. Fatores como exposição à alta temperatura, luz, presença de oxigênio, enzimas e íons metálicos podem influenciar a formação de radicais livres. O radical livre em contato com oxigênio molecular forma um peróxido que, em reação com outra molécula oxidável, induz a formação de hidroperóxido e outro radical livre. As moléculas formadas, contendo o radical livre, ao se romperem formam produtos de peso molecular mais baixo (aldeídos, cetonas, álcoois e ésteres), os quais, segundo Adams (1999), são voláteis e responsáveis pelos odores da rancificação. Tal comportamento pode ser observado para as amostras submetidas aos períodos de 20 e 60 minutos, nas temperaturas de 100 e $150^{\circ} \mathrm{C}$.

Como resultado deste processo, o IPI atinge um valor máximo em algum ponto durante a oxidação térmica, quando a taxa de formação de hidroperóxidos é similar à taxa de decomposição dos mesmos. Após essa etapa, o valor declina gradualmente, como observa-se para os óleos nas condições de exposição à temperatura de $100^{\circ} \mathrm{C}$. Portanto, a medida periódica do índice de peróxido durante a oxidação lipídica permite o reconhecimento desses vários estágios de formação e decomposição. Sendo assim, a aplicação desse índice como fator de avaliação da qualidade do óleo se limita aos estágios iniciais da oxidação, já que quantifica produtos primários da reação, e quanto maior o índice de peróxido inicial do óleo, maior é a fragilidade da amostra às reações de oxidação (White, 2000).

Conforme os dados apresentados nas Tabelas 1 a 4, ao comparar os resultados obtidos de acidez com os valores encontrados para o índice de peróxido, percebe-se que os que possuem uma porcentagem elevada de acidez apresentam níveis de peróxido ainda abaixo do valor máximo determinado pela ANVISA ( $<10 \mathrm{meq} / \mathrm{kg}$ ). Um elevado índice de acidez indica, portanto, que o óleo está sofrendo quebras em sua cadeia, liberando seus constituintes principais: os ácidos graxos (Alves et al., 2009). Este é um indicativo de primeiro estágio de decomposição do óleo, e quando ultrapassa o valor limite, começam a surgir espécies reativas de oxigênio, principalmente peróxidos, substâncias prejudiciais à saúde humana. (Bobbio et al., 2001). Durante a peroxidação é observada uma redução no AGL, acompanhada por alterações organolépticas que revelam a inviabilidade de consumo dos óleos vegetais nessa condição. Para as amostras submetidas ao sobreaquecimento em intervalos de tempo maior, observa-se uma notável redução no índice de iodo se comparado à respectiva amostra controle do óleo (sem aquecimento). Isso se deve à quebra de duplas ligações resultantes de reações de polimerização, ciclização e oxidação, associada com um aumento do ponto de fusão e consistência da amostra (Joseph-Nathan, 1982).

Já a técnica de UV/Vis pode revelar a presença de insaturação em compostos orgânicos, sendo útil para a avaliação da qualidade do óleo. Vários produtos do processo de oxidação térmica mostram uma absorção máxima em comprimentos de onda específicos, como discutido por Vieira e Regitano - 
d'Arce (1999). A oxidação de ácidos poli-insaturados é acompanhada por um aumento de absorção de ultravioletas. Por outro lado, ácidos com insaturação conjugada absorvem majoritariamente na região 230-375 nm (Rovellini et al., 1997), Neste estudo, observou-se um progressivo deslocamento batocrômico do espectro à medida que o estado oxidativo é acentuado com o aumento do tempo de aquecimento e da temperatura das amostras avaliadas. Isto foi observado quando diferentes tipos de óleos vegetais são comparados, sendo o efeito batocrômico pouco expressivo para as amostras analisadas isoladamente. Na Figura 1, é possível se observar os espectros de absorção sobrepostos e a relação de intensidade $\left(\mathrm{I} / \mathrm{I}_{0}\right)$ de amostras controle de óleo de canola, soja, girassol e milho, comparativamente às amostras aquecidas pelo tempo de 10 min variando-se as temperaturas, entre 50 e $150^{\circ} \mathrm{C}$.

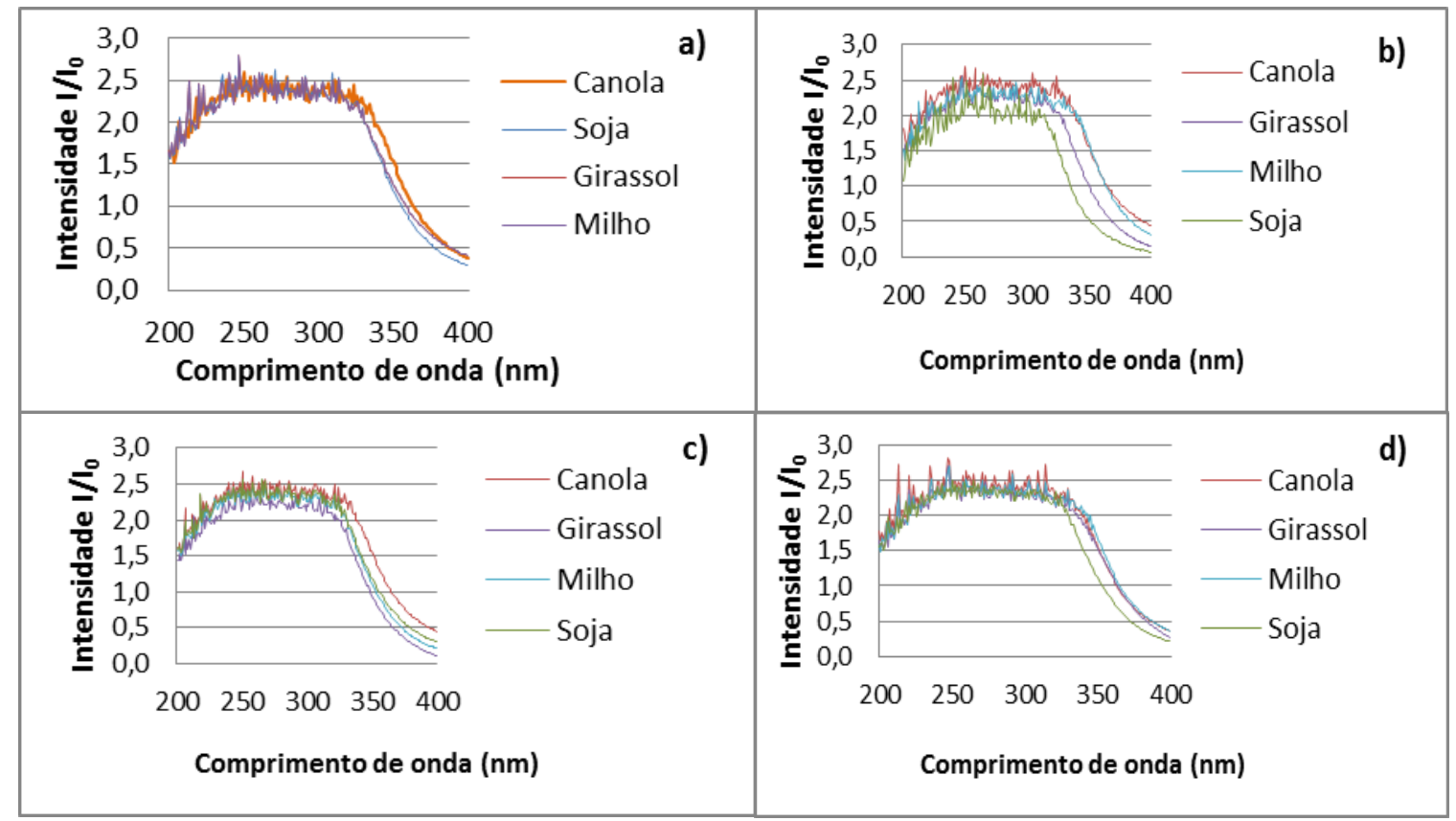

Figura 1 - Relação I/I $\mathrm{I}_{0}$ para diferentes comprimentos de onda de acordo com a variação de temperatura para amostras aquecidas pelo tempo de 10 minutos. (a) Amostras controle $\left(25^{\circ} \mathrm{C}\right)$; (b) Amostras aquecidas a $50^{\circ} \mathrm{C}$; (c) Amostras aquecidas $100^{\circ} \mathrm{C} \mathrm{e} \mathrm{(d)} \mathrm{Amostras} \mathrm{aquecidas} 150^{\circ} \mathrm{C}$.

A análise comparativa da relação entre o tempo de exposição das amostras de óleos a uma determinada temperatura, apresentada na Figura 2, revela um deslocamento batocrômico mais expressivo para as amostras aquecidas pelo tempo de 60 minutos, condição que, segundo as análises físico-químicas e em conformidade com os resultados das Tabelas de 1 a 4 para as condições avaliadas, revelou uma maior atividade termo-oxidativa e comprometimento da qualidade do óleo. Esse resultado é caracterizado pela formação dos radicais livres advindos do processo oxidativo, os quais são constituídos de duplas ligações capazes de absorver na região do ultravioleta. Além disso, vale ressaltar que essas observações são compatíveis com as alterações estruturais que ocorrem nos ácidos graxos livres ou esterificados, com a consequente formação de sistemas conjugados nas reações de peroxidação. 


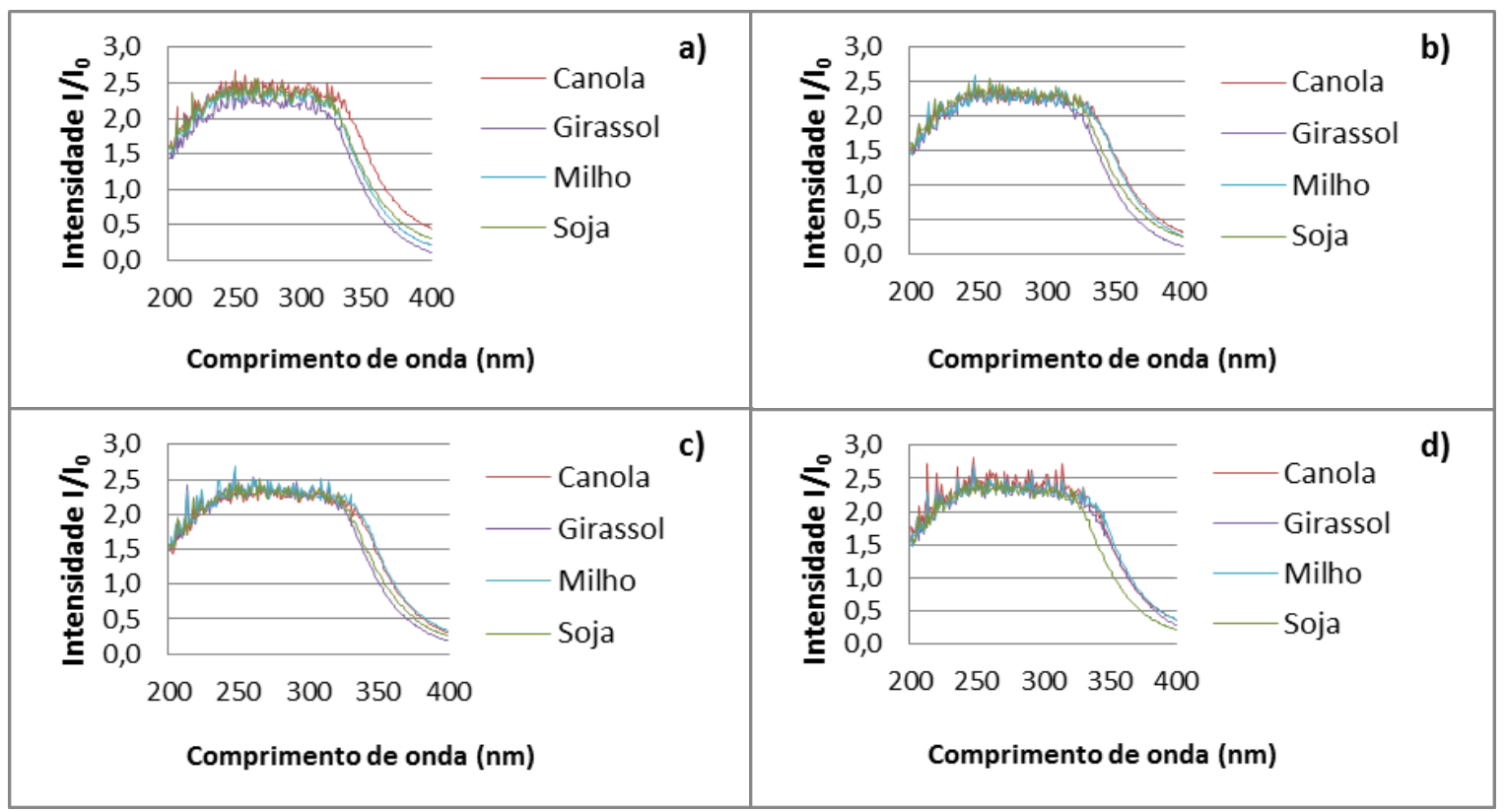

Figura 2 - Relação $\mathrm{I} / \mathrm{I}_{0}$ para diferentes comprimentos de onda de acordo com a variação do tempo para amostras aquecidas a $100^{\circ} \mathrm{C}$. (a) $10 \mathrm{~min}$ (b) $15 \mathrm{~min}$ (c) $20 \mathrm{~min}$ (d) $60 \mathrm{~min}$.

\section{CONCLUSÃO}

A análise de UV/Vis, bem como as análises físico-químicas dos índices de acidez, peróxido e de iodo, demonstraram que à medida que se formam compostos de degradação, a estabilidade oxidativa dos óleos vegetais diminui, comprometendo aspectos químicos e estruturais da composição do óleo e consequentemente a qualidade deste material.

\section{AGRADECIMENTOS} CAPES.

Os autores agradecem o apoio financeiro da FAPEMIG (Processo PCE-00089-14), CNPq e da

\section{REFERÊNCIAS}

ADAMS, C.A. Oxidations and antioxidants. In: Nutricines. Food components in Health and Nutrition.Nottingham Univ. Press. Chapter 2. p.11-34, 1999.

ALMEIDA-DORIA, R.F.; REGITANO-D’ARCE, M.A.B. Antioxidant activity of rosemary and 
oregano ethanol extracts in soybean oil under thermal oxidation. Ciênc. Tec. de Alim., v.20, n.2, p.197-203, 2000.

ALVES, R. F.; GUIMARÃES, S. M.; ABREU T. C.; SILVA, R. D. Índices de Acidez Livre e de Peróxido. Centro de Educação Profissional Hélio Augusto de Sousa, São José dos Campos, SP, 12p., 2009.

AMERICAN OIL CHEMISTS SOCIETY. Official methods and recommended practices of the American Oil Chemists Society. 4th ed. Champaign, USA, AOCS, [AOCS Official method Cd 8-53], 1990.

A.O.C.S, 2004 - AMERICAN OIL CHEMISYS SOCIETY - AOCS - Official Methods and Recommended Practices of the American Oil Chemists Society. Washington, 2004.

BOBBIO, P.A.; BOBBIO, F.O. Química do processamento de alimentos, 3.ed. São Paulo. 33p, 2001.

DAVIS, S.; DARLING-HAMMOND, L.; LAPOINTE, M.; MEYERSON, D. Review of research. School leadership study. Developing successful principals. Palo Alto: Stanford Educational Leadership Institute, 2005.

FARHOOSH, R.; EINAFSHAR, S.; SHARAYEI, P. The effect of comercial refining steps on the rancidity measures of soybean and canola oils. Food Chemistry, Mashhad, n. 115, p.933-938, 2009.

INSTITUTO ADOLFO LUTZ. Normas analíticas do Instituto Adolfo Lutz. v.1.: Métodos Químicos e Físicos para Análise de Alimentos, 3. ed. São Paulo: IMESP, p. 245-246, 1985.

JOSEPH-NATHAN, P. Resonancia Magnetica Nuclear de Hidrogenio-1 y de Carbono-13. Intituto Politécnico Nacional: México, 1982.

MORETTO, E.; FETT, R. Definição de óleos e Gorduras tecnologia de óleos e gorduras vegetais na indústria de alimentos. São Paulo. Editora Varella, 2002

O'BRIEN, R.D.; FARR, W.C.; WAN, P.J. Introduction to fats and oils technology. 2.ed. Champaign: AOCS Press, 2000.

ROVELLINI, P.; CORTESI, N.; FEDELI, E. Ossidazione dei lipid. La Rivista Italiana delle Sostanze Grasse, v. 74, n. 5, p. 181-189, 1997.

SHAHIDI, F.; ZHONG, Y. Lipid Oxidation: Measurement Methods. In: SHAHIDI, Fereidoon. Bailey's Industrial Oil and Fat Products. 6. ed., v.1, Newfoundland: John Wiley \& Sons. Cap. 8, p. 357-386, 2005.

SILVERSTEIN, R. M.; WEBSTER, F. X. Spectrometric Identification of Organic Compounds. 6. ed. New York: John Willey, 1998.

VIEIRA, T. M. F. S.; REGITANO-D'ARCE, M. A. B. UV-Spectrophotometric evaluation of corn oil oxidative stability during microwave heating and oven test.Journal of Agricultural and Food Chemistry, v. 47, n. 6, p. 2003-2006, 1999.

WHITE, P.J. Flavor quality of fats and oils. In: O'BRIEN, R.D., FARR, W.C., WAN, P.J. Introduction to fats and oils technology. 2.ed. Champaign: AOCS Press, 2000. 